\title{
Oridonin Suppresses Proliferation of Human Ovarian Cancer Cells via Blockage of mTOR Signaling
}

\author{
Rong Xia ${ }^{1 \&}$, Sun-Xiao Chen ${ }^{2 \&}$, Qin Qin ${ }^{3 \&}$, Yan Chen ${ }^{3}$, Wei-Wei Zhang ${ }^{3}$, Rong- \\ Rong $\mathrm{Zhu}^{3}$, An-Mei Deng*
}

\begin{abstract}
Oridonin, an ent-kaurane diterpenoid compound isolated from the traditional Chinese herb Rabdosia rubescens, has shown various pharmacological and physiological effects such as anti-tumor, anti-bacterial, and anti-inflammatory properties. However, the effect of oridonin on human ovarian cancer cell lines has not been determined. In this study, we demonstrated that oridonin inhibited ovarian cancer cell proliferation, migration and invasion in a dose-dependent manner. Furthermore, we showed oridonin inhibited tumor growth of ovarian cancer cells (SKOV3) in vivo. We then assessed mechanisms and found that oridonin specifically abrogated the phosphorylation/activation of mTOR signaling. In summary, our results indicate that oridonin is a potential inhibitor of ovarian cancer by blocking the mTOR signaling pathway.
\end{abstract}

Keywords: Oridonin - ovarian cancer - mTOR signal pathway - suppress proliferation

Asian Pac J Cancer Prev, 17 (2), 667-671

\section{Introduction}

Ovarian cancer (OvCa) is the deadliest of all gynecologic cancers; in 2008, more women died from OvCa than from all other gynecologic cancers combined. Each year in the United States, approximately 21,200 women are diagnosed with $\mathrm{OvCa}(2200<45$ years of age $)$, and 14,300 die from the disease $(470<45$ years of age) (Pollack et al., 2009). The standard therapy for advanced epithelial ovarian cancer (EOC) consists of cytoreductive surgery followed by chemotherapy, preferably including paclitaxel combined with cisplatin or carboplatin (Serkies et al., 2011). It is because of the rational use of operation and chemotherapy, that the estimated 5-year relative survival for women diagnosed with OvCa in 2006 was $44.0 \%$, an increase from $36.1 \%$ for those diagnosed between 1975 to 1977(Eheman et al., 2006).But drug toxicity and high price greatly limit the use of these drugs.

A variety of substances, particularly those present in dietary and medicinal plants, are proposed to have an inhibitory effect on OvCa. Oridonin, an ent-kaurane diterpenoid compound (C20H28O6), isolated from the traditional Chinese herb Rabdosia rubescens, has shown various pharmacological and physiological effects, such as anti-tumor, anti-bacterial, and anti-inflammatory properties (Guo et al., 2013; Bu et al., 2014;Wang et al., 2014). However, the effect of oridonin on human OvCa cell lines has not been determined. Therefore, the present study investigated the effect of oridonin on the migration and invasion of the OvCa cell line and the underlying molecular mechanism involved.

\section{Materials and Methods}

\section{Cell lines, cell culture and reagents}

Oridonin was purchased from Sigma-Aldrich (USA). A $10 \mathrm{mM}$ solution of oridonin was prepared in sterile water, stored at $-20^{\circ} \mathrm{C}$ and protected from light, and diluted to needed concentrations for studies. Human ovarian cancer cells (SKOV3,OVCAR-3 and A2780) were purchased from the American Type Culture Collection (ATCC, USA), cultured and maintained in Dulbecco's modified Eagle's medium (DMEM; Hyclone, USA), supplemented with $10 \%$ heated-inactivated fetal calf serum (FCS), 100 $\mathrm{U} / \mathrm{mL}$ penicillin, $10 \mu \mathrm{g} / \mathrm{mL}$ streptomycin sulfate and then held at $37^{\circ} \mathrm{C}$ in a humidified $5 \% \mathrm{CO}_{2}$ incubator. Mitomycin $\mathrm{C}$ was ordered from Roche (China).

\section{Cell viability assay}

Human ovarian cancer cells $\left(2 * 10^{4}\right.$ cells/well $)$ were treated without or with different concentrations of oridonin for $48 \mathrm{~h}$. Cell viability was determined by MTT(SigmaAldrich, USA) with Bio-Radmicroplate reader.

\section{Flow cytometry analysis}

Human ovarian cancer cells $\left(1^{*} 10^{6}\right)$ were treated with different concentrations of oridonin for $48 \mathrm{~h}$ and then collected and analyzed for cell cycle distribution in

${ }^{1}$ Department of Transfusion, Huashan Hospital, Fudan University, ${ }^{2}$ Changzheng Hospital, Second Military Medical University, ${ }^{3}$ Department of Laboratory Diagnosis, Changhai Hospital, Second Military Medical University, Shanghai, P. R. China ${ }^{\circledR}$ Equal contributors*For correspondence: amdeng70@163.com 
a FACS flow cytometer (BD Sciences, USA) with cell cycle Kit (BD Pharmingen, USA).

\section{Wound healing assay}

Human ovarian cancer cells were cultured into full confluence in 6-well plates and then were incubated with $10 \mu \mathrm{g} / \mathrm{mL}$ mitomycin $\mathrm{C}$ for 2 hours to inactivate cell proliferation. Cells were washed with PBS and wounds were made by sterile pipette tips. DMEM supplemented with $0.5 \%$ FBS was added into wells with or without different concentrations of oridonin. Cell images were taken after 10 hours of incubation with the aforementioned reagents.

\section{Transwell migration assay}

The transwell (Corning Incorporated, USA) were coated with 50 $\mu \mathrm{l}$ 1:8 diluted Matrigel (Growth factor reduced, BD Biosciences, USA) for $30 \mathrm{~min}$ in cell incubator. The bottom chambers of transwell were filled with DMEM with $0.5 \%$ FBS and the top chambers were seeded with mitomycin $\mathrm{C}$ inactivated, $4 \times 10^{4}$ cells/well in $100 \mu \mathrm{L}$ DMEM $(0.5 \%$ FBS $)$ plus different concentrations of oridonin. After overnight, the cells on the top surface of the membrane (non-migrated cells) were scraped with a cotton swab and the cells migrated onto the bottom sides of the membrane (invasive cells) were fixed with cold $4 \%$ paraformaldehyde and stained with crystal Violet. Images were taken using OLYPUS inverted microscope.

\section{Xenograft mouse model}

Xenograft mouse model was used to evaluate the effect of oridonin tumor growth as described by $\mathrm{Yi}$ et al (Yi et al., 2008). The 5-week-old to 6-week-old severe combined immune deficiency (SCID) male mice (SLAC Laboratory Animal, China) weighing about $20 \mathrm{~g}$ were divided into four groups with 6 animals for each group: control group, 5-FU group, oridonin high-dose treated group and oridonin lowdose treated group. SKOV 3 cells, $2 \times 10^{6}$ cells per mouse, were s.c. injected into the mice. After the tumors had established (about $100 \mathrm{~mm} 3$ ), oridonin dissolved in sterile PBS, was administered by intraperitoneal injection at 5 or $10 \mathrm{mg} / \mathrm{kg}$ body weight, respectively. $5-\mathrm{FU}$, dissolved in sterile PBS, was administered by intraperitoneal injectionat $20-\mathrm{mg} / \mathrm{kg} /$ two days body. Control animal was only given $0.2 \mathrm{ml}$ sterile water as vehicle control. The mouse body weight and tumor sizes were recorded every two days and the tumor sizes were determined by Vernier caliper measurements and calculated as $1 / 2 \times$ length $\times$ width $\times$ height. After 21 days, mice were sacrificed. Animals used in the present study were approved by the Ethics Committee of Shanghai University of Traditional Chinese Medicine where the work was undertaken and that it conforms to the provisions of the Declaration of Helsinki in 1995.

\section{Western blotting}

To determine the effects of oridonin on blocking the phosphorylation of mTOR signaling pathway, cells were first starved with $0.1 \%$ FBS medium for 12-14 hours. After washout with new fresh culture medium, cells were pretreated with different concentrations of oridonin for
60 minutes before the phosphorylation status of mTOR signaling molecules was determined. The whole cell extracts were prepared by lysing cells with RIPA buffer supplemented with proteinase inhibitors. Antibodies used for different Western blot analyses, are anti-phosphomTOR (Ser2448), phospho-4EBP1 (Thr37/46) mAb and phospho-P70S6 (Thr389) (Cell Signaling Technology, USA).

\section{Statistical analysis}

The data are presented as mean $\pm \mathrm{SE}$, and statistical comparisons between groups were performed using T-test. $P$ value $\leq 0.05$ was considered significance statistically.

\section{Results}

\section{Inhibition of Ovarian cancer cell lines proliferation by oridonin}

Oridonin is a diterpenoid purified from Rabdosia rubescens and has a molecular weight of $364.43 \mathrm{~g} / \mathrm{mol}$ (Figure 1a). We first tested the in vitro anti-proliferative effect of oridonin on Ovarian cancer cell lines SKOV3, OVCAR-3 and A2780 using a standard proliferation assay (MTT). Our results showed that oridonin significantly reduced SKOV3, OVCAR-3 and A2780 cell proliferation at the IC50 of $17.21 \mu \mathrm{M}, 13.9 \mu \mathrm{M}$ and $12.1 \mu \mathrm{M}$, respectively (Figure 1b, c, d). These data suggest that oridonin preferentially be an anti-OvCa reagent. Further, we examine whether oridonin affected cell cycle progression and apoptosis and our results revealed that it induced a

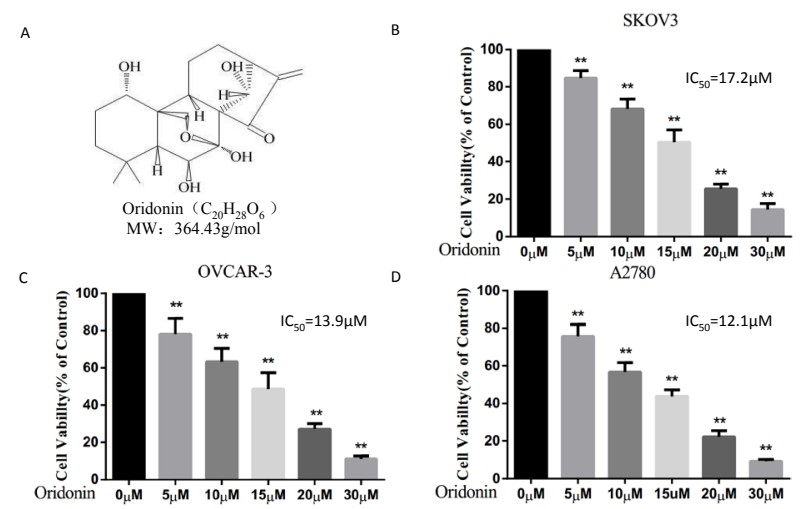

Figure 1. Oridonin Inhibits Cell Viability in OvCa SKOV3, OVCAR-3 and A2780 Cell. (a) The chemical structure of oridonin with a molecular weight $364.43 \mathrm{~g} / \mathrm{mol}$. (b) Oridonin inhibits SKOV3 cell viability in dose-dependent manner. SKOV 3 cell $(2 * 104$ cells/well) were starved with $0.1 \%$ fetal bovine serum (FBS) medium and then treated with or without different concentrations of oridonin for $24 \mathrm{~h}$. Cell viability was quantified by MTT assay $(* P<0.05, * * P<0.01$ versus Control). (c) Oridonin inhibits OVCAR-3 cell viability in dose-dependent manner. OVCAR-3 cell $\left(2 * 10^{4}\right.$ cells $/$ well $)$ were starved with $0.1 \%$ fetal bovine serum (FBS) medium and then treated with or without different concentrations of oridonin for $24 \mathrm{~h}$. Cell viability was quantified by MTT assay $(* P<0.05, * * P<$ 0.01versus Control). (d) Oridonin inhibits A2780 cell viability in dose-dependent manner. A2780 cell $\left(2 * 10^{4}\right.$ cells $/$ well $)$ were starved with $0.1 \%$ fetal bovine serum (FBS) medium and then treated with or without different concentrations of oridonin for $24 \mathrm{~h}$. Cell viability was quantified by MTT assay $\left({ }^{*} P<0.05\right.$, $* * P<0.01$ versus Control). Column, mean from three different experiments with six duplicates; bar, SE 
G2M phase block, $44.71 \%$ treated vs. $19.21 \%$ control and a decrease of cells in G1/M phase, from $57.43 \%$ to $36.02 \%$ (Table 1).

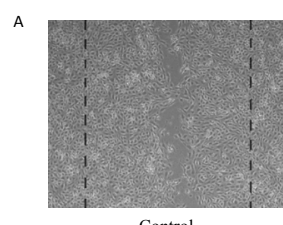

Control

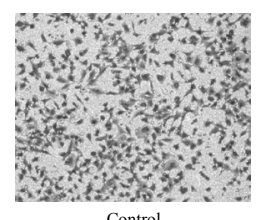

$10 \mu \mathrm{M}$

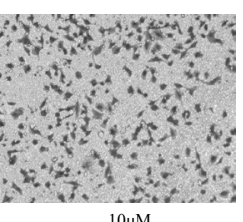

$10 \mu \mathrm{M}$

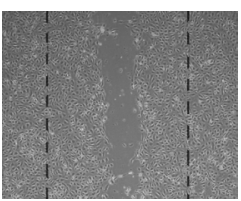

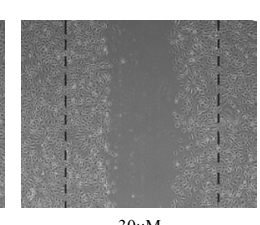
$30 \mu \mathrm{M}$

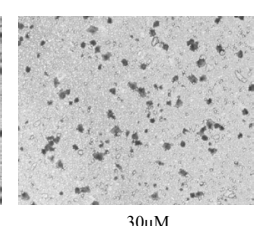

Figure 2. Oridonin Inhibits Migration, Invasion of SKOV3 Cells. (a) Oridonin inhibits SKOV3 cells migration. KOV3 cells were allowed to grow into full confluence in six-well plates, and inactivated with $10 \mathrm{lg} / \mathrm{mL}$ mitomycin $\mathrm{C}$ for $2 \mathrm{~h}$. Cells were wounded with pipette and treated with or without different concentration of oridonin in endothelial cell medium supplemented with $0.5 \%$ fetal bovine serum (FBS). After incubation, the migrated cells were quantified by manual counting. (b) Oridonin inhibits the invasion of KOV3 cells. KOV3 cells were seeded in the upper chamber of transwell and treated with different concentrations of oridonin. After about 8-10 h, the invasive KOV3 cells passed through the membrane and were quantified by counting the cells that migrated onto the membrane. Column, mean from three different experiments with duplicates; bar, $\mathrm{SE}(* P<0.05, * * P<0.01$ versus Control)
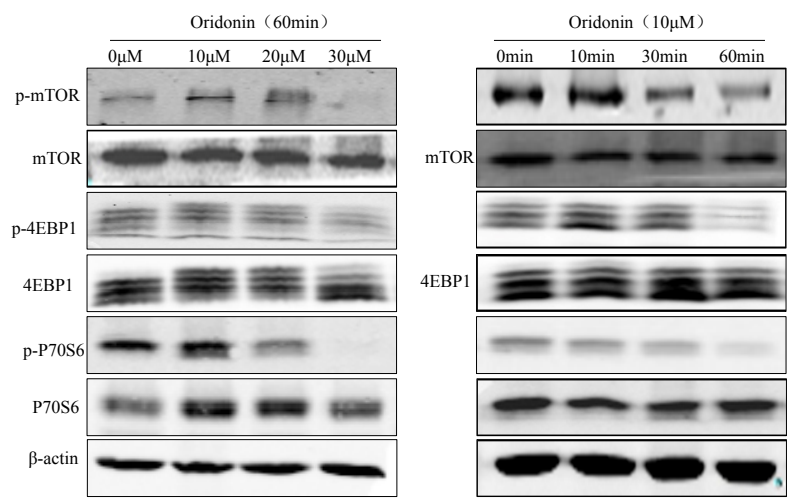

Figure 4. Oridonin Inhibits the mTOR Signaling Pathway in SKOV Cells. (a) Effects of oridonin on blocking the phosphorylation of mTOR signaling pathway. SKOV3 cells were pretreated with various concentrations of oridonin for $60 \mathrm{~min}$. After that, cells were washed with cold phosphatebuffered saline (PBS) and lysed on the dish in RIPA buffer. Phosphorylation and activation of different protein kinases including pSER2248-mTOR, p Thr37/46-4EBP1 and pThr389P70S6 were examined by specific antibodies.(b) A time course of SKOV3 cells without or with the presence of oridonin $(30 \mu \mathrm{M})$ : $0,10,30,60 \mathrm{~min}$
Oridonin inhibits migration, invasion of SKOV3 cells

Tumor migration and invasion are the essential steps in tumorigenesis(Miao et al., 2014). We determined the effects of oridonin on the chemotactic motility of SKOV3 cells using wound-healing migration and transwell cell invasion assays. As shown in Fig2 (a, b), Oridonin significantly inhibited SKOV3 cells migration and invasion at $10 \mu \mathrm{M}$. Moreover, the inhibition of oridonin on the migration of SKOV3 cells were in a dose-dependent manner.

\section{Oridonin inhibits tumor growth in vivo}

We tested oridonin's in vivo effect in a xenograft human ovarian cancer model. Tumor-bearing mice had been treated by oridonin or 5-FU for 21 days. As shown in Fig 3a, tumors in control animals grew rapidly (from $201.59 \pm 34.67$ to $1494.56 \pm 189.77 \mathrm{~mm} 3$ ), whereas tumors in oridonin-treated animals grew much slower (from $132.45 \pm 18.47$ to $653.12 \pm 75.87 \mathrm{~mm} 3$ ). Mice treated with oridonin did not have altered body weight (Figure 3b), suggesting no toxicity of the compound at the tested concentration.

Oridonin inhibits the mTOR signaling pathway in SKOV cells

mTOR pathway is a crucial mediator of tumor progression (Zagouri et al., 2012). To examine the
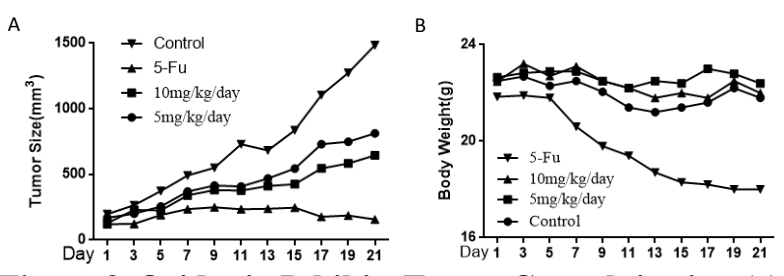

Figure 3. Oridonin Inhibits Tumor Growth in vivo. (a) Oridonin inhibits solid cancer growth in xenograft ovarian cancer mouse model. SKOV 3 cells were injected $\left(2 * 10^{6}\right.$ per mouse) into the 5- to 6-week-old male nude mice. After the tumors had established (about $100 \mathrm{~mm} 3$ ), the mice were injected with or without oridonin at 5 or 10 $\mathrm{mg} / \mathrm{kg}$ every day, respectively. After 21 days, mice were sacrificed, tumors were removed and photographed. Tumor sizes in control animals and oridonin treated animals were calculated and shown in (a). Tumors in control animals increased from $201.59 \pm 34.67$ to $1494.56 \pm 189.77 \mathrm{~mm}^{3}$, whereas tumors in animals treated with oridonin at dose of $10 \mathrm{mg} / \mathrm{kg}$ per day increased from $132.45 \pm 18.47$ to $653.12 \pm 75.87 \mathrm{~mm}^{3}$. (b) Treatment with oridonin has little effect on mouse body weight. No significant difference between animals treated with oridonin at dose of $10 \mathrm{mg}$ / $\mathrm{kg}$ per day and the control animals. Column, mean; bar, $\mathrm{SE}(\mathrm{n}=7)$

Table 1. The Cell Cycle and Apoptosis of SKOV3 after Transfection and Interference by Using Flow Cytometry

\begin{tabular}{llcccc}
\hline Group & $\mathrm{n}$ & $\mathrm{G} 0 / \mathrm{G} 1(\%)$ & $\mathrm{S}(\%)$ & $\mathrm{G} 2 / \mathrm{M}(\%)$ & Apoptosis(\%) \\
\hline Control & 7 & $57.43 \pm 6.23$ & $23.36 \pm 3.49$ & $19.21 \pm 2.97$ & $1.23 \pm 0.21$ \\
30uM & 7 & $36.02 \pm 5.39 * *$ & $16.11 \pm 1.45$ & $44.71 \pm 3.02^{* *}$ & $43.47 \pm 8.46^{* *}$ \\
\hline
\end{tabular}

\footnotetext{
${ }^{\#}$ Compared with control $P<0.01$
} 
underlying molecular mechanism of oridonin's inhibition on proliferation, we studied the effects of oridonin on the phosphorylation of mTOR signaling in SKOV3 cells. Our results showed that oridonin at concentration of $30 \mu \mathrm{M}$ significantly inhibited the phosphorylation of protein kinases involved in the mTOR signaling pathway, including mTOR, 4EBP1, and P70S6 (Figure 4a). We also studied a time course of SKOV3 without or with the presence of oridonin $(30 \mu \mathrm{M}): 0,10,30,60 \mathrm{~min}$. The results showed that the oridonin got to inhibit the phosphorylation of mTOR signaling at $30 \mathrm{~min}$ (Figure 4b).

\section{Discussion}

Looking for small molecules which have anti-tumor ability in natural Chinese herbal medicine has been the focus of cancer drug development (Konkimalla et al., 2008;Assaf et al., 2013). In this report, we found that oridonin, an ent-kaurane diterpenoid compound, which has long been used as an anti-inflammation drug, inhibited OvCa tumorigenesis. To further understand the mechanisms in the anti-OvCa activity of oridonin, we analyzed the effect of oridonin on mTOR signaling pathways which were constitutively activated in SKOV3 ovarian cells. It has been shown that oridonin induces apoptosis and attenuates the migration and invasion of melanoma cells and breast cancer cells, as well as decreasing the expression of Bcl-2,caspase-8,NF- $x \mathrm{~B}$ and PARP proteins(Ren et al., 2006; Wang et al., 2013). Our current studies showed that oridonin repressed SKOV3 cells migration and invasion through inhibiting mTOR signaling pathway. In a xenografts mouse model, we found that oridonin $(10 \mathrm{mg} / \mathrm{kg})$ did not affect the body weight of the mice, while exhibited significant inhibitory effects on OvCa solid tumor growth.

As we knew, mTOR is directly involved in many cell signaling pathways and many aberrations of the mTOR implicated in human cancer. For example, PI3K amplification/mutation, AKT overexpression, loss of PTEN and P53 function, and overexpression of S6K1 have all been associated with cancer development and are linked to the mTOR signaling pathway(Li et al., 2012; Wang et al., 2014;Xu et al., 2014;Zhang et al., 2014;Zhao et al., 2014). Although mutations of mTOR itself have not been reported, mutations in components of mTOR-related signaling pathways have frequently been described in various human malignancies. Notably, tumor cells with mutations in p53 or PTEN, found in more than $50 \%$ of human tumors, suggesting that inhibition of mTOR signaling might be a potential tumor-selective therapeutic strategy(Husseinzadeh et al., 2014). We found that oridonin inhibited the phosphorylation of mTOR in SKOV3 ovarian cells, but did not affect the levels of total mTOR proteins. The reduction of phosphorylated mTOR might explain the observed decrease of the phosphorylation of 4EBP1 and P70S6, the major downstream target of MTOR, following treatment with oridonin. One possible mechanism of how oridonin decreases the phosphorylation of mTOR was that oridonin was directly binding to the kinase domain of mTOR. Our results illustrated that oridonin could significantly inhibit the activation of mTOR signaling pathway in a concentration-dependent manner with a modest, effective concentration of $30 \mu \mathrm{M}$.

In summary, this study shows that oridonin inhibits the phosphorylation of mTOR signaling pathway in vitro and tumor tumorigenesis in vivo and is efficacious against the xenograft model of human ovarian cancer in nude mice. Taken together, our studies indicate that oridonin is a potential inhibitor of tumor migration and invasion by blocking mTOR signaling pathway.

\section{Acknowledgements}

This research was supported by grants from 973 Foundation (2013CB531606), National Science Foundation of China $(81471605,81472770,81272280$, 81273282 , 81202353, 81371786, 81302579), Shanghai Municipal Commission for Science and Technology (11JC1410902), Changhai Hospital (CH125530300), Grant of Nanjing District (12MA056)

\section{References}

Assaf AM, Haddadin RN, Aldouri NA, et al (2013). Anti-cancer, anti-inflammatory and anti-microbial activities of plant extracts used against hematological tumors in traditional medicine of Jordan. J Ethnopharmacol, 145, 728-36.

Bu HQ, Liu DL, Wei WT, et al (2014). Oridonin induces apoptosis in SW1990 pancreatic cancer cells via p53- and caspase-dependent induction of p38 MAPK. Oncol Rep, 31, 975-82.

Eheman CR, Peipins L, Wynn M, et al (2006). Development of a public health research program for ovarian cancer. $J$ Womens Health (Larchmt), 15, 339-45.

Guo W, Zheng $P$, Zhang J, et al (2013). Oridonin suppresses transplant rejection by depleting $\mathrm{T}$ cells from the periphery. Int Immunopharmacol, 17, 1148-54.

Husseinzadeh N and Husseinzadeh HD (2014). mTOR inhibitors and their clinical application in cervical, endometrial and ovarian cancers: A critical review. Gynecol Oncol,

Konkimalla VB and Efferth T (2008). Anti-cancer natural product library from traditional chinese medicine. Comb Chem High Throughput Screen, 11,7-15.

Li CY, Huang WF, Wang QL, et al (2012). Crocetin induces cytotoxicity in colon cancer cells via p53-independent mechanisms. Asian Pac J Cancer Prev, 13, 3757-61.

Miao L, Xiong X, Lin Y, et al (2014). miR-203 inhibits tumor cell migration and invasion via caveolin-1 in pancreatic cancer cells. Oncol Lett, 7, 658-62.

Pollack LA, Rowland JH, Crammer C, et al (2009). Introduction: charting the landscape of cancer survivors' health-related outcomes and care. Cancer, 115, 4265-9.

Ren KK, Wang HZ, Xie LP, et al (2006). The effects of oridonin on cell growth, cell cycle, cell migration and differentiation in melanoma cells. J Ethnopharmacol, 103, 176-80.

Serkies K, Wegrzynowicz E and Jassem J (2011). Paclitaxel and cisplatin chemotherapy for ovarian cancer during pregnancy: case report and review of the literature. Arch Gynecol Obstet, 283, 97-100.

Wang CD, Yuan CF, Bu YQ, et al (2014). Fangchinoline inhibits cell proliferation via Akt/GSK-3beta/ cyclin D1 signaling and induces apoptosis in MDA-MB-231 breast cancer cells. Asian Pac J Cancer Prev, 15, 769-73.

Wang H, Ye Y and Yu ZL (2014). Proteomic and functional analyses demonstrate the involvement of oxidative stress in the anticancer activities of oridonin in HepG2 cells. Oncol 
Rep, 31, 2165-72

Wang S, Zhong Z, Wan J, et al (2013). Oridonin induces apoptosis, inhibits migration and invasion on highlymetastatic human breast cancer cells. Am J Chin Med, 41,177-96.

Xu WT, Yang Z, Lu NH (2014). Roles of PTEN (phosphatase and tensin homolog) in gastric cancer development and progression. Asian Pac J Cancer Prev, 15, 17-24.

Yi T, Yi Z, Cho SG, et al (2008). Gambogic acid inhibits angiogenesis and prostate tumor growth by suppressing vascular endothelial growth factor receptor 2 signaling. Cancer Res, 68, 1843-50.

Zagouri F, Sergentanis TN, Chrysikos D, et al (2012). mTOR inhibitors in breast cancer: a systematic review. Gynecol Oncol, 127, 662-72.

Zhang T, Qu S, Shi Q, et al (2014). Evodiamine induces apoptosis and enhances TRAIL-induced apoptosis in human bladder cancer cells through mTOR/S6K1-mediated downregulation of Mcl-1. Int J Mol Sci, 15, 3154-71.

Zhao H, Wang L, Wei R, et al (2014). Activation of glucagon-like peptide-1 receptor inhibits tumourigenicity and metastasis of human pancreatic cancer cells via PI3K/Akt pathway. Diabetes Obes Metab, 16, 850-60 